\title{
EVALUACIÓN EN LÍNEA: FORTALEZAS Y DEBILIDADES DE LA EVALUACIÓN EN LÍNEA DESDE LA PERCEPCIÓN DE ESTUDIANTES Y PROFESORES DE LA CARRERA DE TRABAJO SOCIAL*1
}

\author{
ONLINE EVALUATION: STRENGTHS AND WEAKNESSES OF \\ ONLINE EVALUATION FROM THE PERCEPTION OF \\ STUDENTS AND PROFESSORS OF THE SOCIAL WORK CAREER
}

\author{
Ángela Venegas Meza ${ }^{2}$ \& Ana María Galdames Paredes ${ }^{3}$ \\ angela.venegas@ucentral.cl - ana.galdames@ucentral.cl \\ Universidad Central de Chile \\ Santiago, Chile
}

\section{RESUMEN}

Considerando la importancia de la evaluación dentro del proceso de enseñanza aprendizaje, las posibilidades formativas que otorga la tecnología a través de las plataformas educativas virtuales y la baja utilización de la plataforma virtual que dispone la Universidad Central de Chile, se implementó un proyecto de innovación educativa destinado a identificar fortalezas y debilidades en la aplicación de evaluaciones en línea de una asignatura disciplinar correspondiente al primer semestre de la carrera de Trabajo Social, considerando un total de 25 estudiantes, ajustando dicha evaluación a los objetivos de aprendizaje establecidos para tal asignatura, a través de una metodología mixta que recogió datos cuantitativos mediante una encuesta, mientras que, desde lo cualitativo, se realizó un grupo focal.

Los resultados arrojaron como fortalezas la posibilidad de contar con retroalimentación inmediata acerca del avance de los aprendizajes obtenidos, lo que incide favorablemente en la motivación de los y las estudiantes, así como la fácil aplicabilidad de ciertos instrumentos evaluativos, y la posibilidad de entrenar el uso de las TICs en el escenario docente actual.

Entre las debilidades, se identificó el mayor tiempo que implica el diseño instruccional y validación de los instrumentos evaluativos para el equipo docente, la escasa preparación de los docentes respecto al uso de todas las aplicaciones del aula virtual.

Los hallazgos de la implementación del proyecto de innovación educativa se constituyen en un aporte al trabajo del comité curricular de la carrera, ya que deja en evidencia las posibilidades de réplica en los distintos niveles por su incidencia positiva en la motivación del estudiante y al mismo tiempo dan cuenta de la necesidad de generar instancias formativas que tributen al desarrollo de competencias digitales tanto en profesores y especialmente en estudiantes con la

\footnotetext{
* Artículo recibido el 23 de enero de 2019; aceptado el 28 de marzo de 2019.

1 Este artículo correpsonde al proyecto de innovación educativa sobre evaluaciones en línea, en que participan ambas autoras.

${ }^{2}$ Magíster en Intervención Social, Diplomada en Prevención del Consumo Problemático de Drogas y Alcohol en Establecimientos Educacionales y Diplomada en Innovación Didáctica a la Docencia Universitaria. Trabajadora Social.

3 Doctora en Educación, Magíster en Pedagogía Universitaria, Trabajadora Social y Diplomada en mediación familiar y escolar. Ha integrado equipos interdisciplinarios, en el ámbito familiar y educacional, desarrollando conjuntamente docencia universitaria en instituciones de Educación Superior.
} 
finalidad de desarrollar una actitud positiva, crítica y realista respecto de las fortalezas y debilidades del uso de la tecnología en el proceso de enseñanza aprendizaje en el actual contexto de aprendizaje en la sociedad del conocimiento y la era digital en dónde el acceso a los diversos beneficios de la política social a los cuales se accedía de manera presencial se ha trasladado a plataformas virtuales.

Así mismo, este artículo aporta a la reflexión de la evaluación en línea implementada en la educación superior, desde la perspectiva de profesores y estudiantes, que puede facilitar su ajuste y desarrollo en otros contextos similares.

Palabras claves: evaluación en línea, plataforma virtual, educación superior, Trabajo Social.

\section{ABSTRACT}

Considering the importance of evaluation within the teaching-learning process, the training possibilities provided by technology through virtual educational platforms and the low use of the virtual platform available at the Central University of Chile, an educational innovation project was implemented. aimed at identifying strengths and weaknesses in the application of online evaluations of a disciplinary subject corresponding to the first semester of the Social Work career, considering a total of 25 students, adjusting said evaluation to the learning objectives established for such subject, through of a mixed methodology that collected quantitative data through a survey, while, from the qualitative, a focus group was carried out.

The results showed as strengths the possibility of having immediate feedback on the progress of the learning obtained, which has a favorable impact on the motivation of the students, as well as the easy applicability of certain evaluative instruments, and the possibility of training the use of ICTs in the current teaching scenario.

Among the weaknesses, the longer time involved in the instructional design and validation of the evaluation instruments for the teaching team, the poor preparation of the teachers regarding the use of all the applications of the virtual classroom was identified.

The findings of the implementation of the educational innovation project constitute a contribution to the work of the curricular committee of the career, since it reveals the possibilities of replication at different levels due to its positive impact on student motivation and at the same time realize the need to generate training instances that contribute to the development of digital skills both in teachers and especially in students in order to develop a positive, critical and realistic attitude regarding the strengths and weaknesses of the use of technology in the process of teaching learning in the current context of learning in the knowledge society and the digital age where access to the various benefits of social policy that were accessed in person has been transferred to virtual platforms.

Likewise, this article contributes to the reflection of the online assessment implemented in higher education, from the perspective of teachers and students, which can facilitate its adjustment and development in other similar contexts.

Key words: Online assessment, virtual platform, higher education, Social Work. 


\section{Introducción}

El presente artículo detalla la implementación de un proyecto de innovación educativa destinado a identificar fortalezas y debilidades de la implementación de evaluaciones en línea según la percepción de estudiantes de primer año y profesores de la carrera de Trabajo Social, de la Universidad Central de Chile.

Los fundamentos pedagógicos para el desarrollo de esta innovación en la evaluación de aprendizajes radican en que por un lado asistimos a una época de cambios sustantivos en los modelos pedagógicos en educación superior, en tanto se modifica el foco desde la enseñanza al aprendizaje lo cual pone en el centro del proceso al estudiante, generando una nueva cultura de la evaluación concebida como una instancia más de aprendizaje, esto implica que el estudiante aprende según cómo es evaluado, lo que justifica el uso de diversas metodologías de evaluación para promover diversos saberes favoreciendo la implicación de los estudiantes, y, por otro lado, nos encontramos viviendo en la sociedad del conocimiento en la que tanto el acceso como uso de las TICs son un requisito para la participación efectiva en una sociedad que ha trasladado a una plataforma virtual una serie de servicios de la política social a los que hace un par de años atrás sólo se podía acceder de manera presencial.

En este sentido es fundamental incorporar en el proceso de enseñanza aprendizaje el uso de las TICs para aproximarlos a las nuevas formas de recogida y procesamiento de información en fuentes digitales y la utilización de dichas tecnologías como herramientas de investigación social y al mismo tiempo como un recurso de comunicación y gestión de la información dado que este es el escenario en el cual se desarrolla la intervención social en los diversos ámbitos del quehacer disciplinar en la actualidad, y el no saber manejar dichos sistemas y plataformas online puede convertirse en un factor de exclusión profesional de los futuros y futuras profesionales del Trabajo Social.

Así mismo familiarizar a los estudiantes con el uso de TICs se relaciona con el compromiso de la universidad respecto de formar estudiantes bajo un enfoque de competencias lo que implica que se trabajen programas de estudio en estrecho contacto con el mundo laboral e incorporar la práctica temprana y del "saber hacer" como un elemento central del currículo.

Una de las principales características de la sociedad del conocimiento es la inmediatez en el acceso a la información, inmediatez a la que las juventudes actuales están acostumbradas pues en tanto la mayoría son nativos digitales han crecido con la lógica de la conectividad instantánea a tan sólo un clic de distancia. Esta es una de las grandes motivaciones que mantiene a jóvenes reunidos en comunidades de juegos en línea en un espacio donde las fronteras geográficas no existen, dichos juegos ofrecen la posibilidad de jugar en tiempo real y de compartir una experiencia de entretención sin salir de sus casas.

Así entonces se trabaja en equipo para lograr los objetivos de los juegos online y eso mantiene permanentemente la motivación de los participantes quienes acceden a diversos escenarios de juego según los intereses pudiendo luchar contra monstruos de la prehistoria o zombis del futuro. La posibilidad de acceder a múltiples y diversos escenarios virtuales que superan a la realidad en cuanto a atractivo e información es una de las principales razones que mantiene a un sector de las juventudes en permanente consumo de los escenarios que se han posicionado fuertemente desde el espacio del ocio, la entretención y el intercambio de relaciones sociales. En contraposición en el ámbito educativo universitario los docentes disponen de salas de clases con una infraestructura clásica, tradicional, ambientadas con colores neutros y estandarizados, con pizarras blancas para desarrollar una clase intentando captar la atención y mantener la motivación de estas juventudes estudiantes acostumbradas a recibir y procesar información 
diversa y de manera simultánea, al mismo tiempo que experimentan múltiples estímulos desde sus dispositivos electrónicos, pudiendo escuchar música y al mismo tiempo estar comentando el estado de un amigo en Instagram y participar de una conversación en un grupo de WhatsApp.

En este sentido la sala de clases en la educación superior sigue manteniendo un formato tradicional en cuanto a su disposición física, la mayoría de los docentes siguen utilizando métodos tradicionales tanto en el proceso de enseñanza aprendizaje como en el proceso evaluativo, cuyo principal material didáctico generalmente se reduce a presentaciones de diapositivas mediante programa de presentación Power point del conjunto de herramientas de Microsoft Office.

Esta lógica de educación tradicional en la educación superior se evidencia no sólo en el tipo de material didáctico, sino que se visualiza especialmente en los procesos de evaluación que se siguen utilizando, como las pruebas de cátedra escritas en las que el docente diseña un instrumento evaluativo en papel, el cual es entregado al curso en la fecha previamente establecida y cada estudiante responde dicho instrumento cuyas respuestas se consignan mediante escritura a mano y luego el docente dispone de una a dos semanas para su respectiva corrección y calificación.

Si bien los estudiantes saben y conocen de ese tiempo para la revisión y calificación de sus pruebas y trabajos, es frecuente que a la clase siguiente de haber realizado la prueba ellos pregunten la fecha de entrega de resultados, lo que da cuenta de la importancia de la retroalimentación oportuna de sus aprendizajes porque a través de ella no sólo se enteran de aquello que hicieron o respondieron mal sino que además acumulan saberes que les ayudarán a enfrentar con mayor preparación evaluaciones futuras.

Esto obedece a que la retroalimentación oportuna y de calidad, sin duda favorece la motivación del estudiante respecto de su proceso educativo y respecto de la asignatura.

Con todos estos antecedentes es que desde el Comité Curricular de la carrera de Trabajo Social surge la iniciativa de diseñar un proceso de evaluación que por un lado familiarizara a los estudiantes con el uso de TICs entendida como habilidad fundamental para el actual mundo laboral y que al mismo tiempo les permitiera contar con una retroalimentación inmediata.

Así surge el proyecto de innovar respecto del diseño y formato de las evaluaciones en la cátedra de Pobreza y Exclusión Social ubicada en el primer semestre de la carrera con la finalidad de analizar ¿Cuáles son las fortalezas y debilidades de la implementación de evaluaciones en línea según la percepción de estudiantes de primer año y profesores de la carrera de Trabajo Social?

En este contexto se implementó a partir de marzo del año 2019 en la cátedra mencionada una metodología mixta en la que las clases se desarrollaron $100 \%$ de manera presencial y las tres evaluaciones de contenido se llevaron a cabo en línea a través de la plataforma Moodle.

\subsection{Discusión Bibliográfica}

\subsubsection{Sociedad del conocimiento}

Diversos autores señalan que hoy en día la tecnología forma parte fundamental de la vida de las personas y está presente tanto en la mayoría de los espacios familiares, como profesionales y sociales; "desde una tableta o un teléfono móvil, hasta los equipos médicos o de laboratorio, pasando por los códigos de barra de los productos que se comercializan en casi 
cualquier establecimiento 0 las transacciones bancarias efectuadas mediante cajeros electrónicos, en todo se hallan las tecnologías nuevas" (Hernández et al. 2016 p. 226). En este contexto, los autores reflexionan respecto a la ardua tarea de la labor educativa en cuanto al desafío de desarrollar múltiples competencias y experiencias de aprendizaje significativas que contribuyan con el establecimiento de una cultura digital entre los estudiantes.

Bajo el precepto de que, sin la formación continua requerida para acceder al mundo digital, difícilmente los estudiantes (en tanto sujetos) tendrán la capacidad de participar activamente de esta sociedad.

Asistimos a un nuevo tipo de civilización de la información que gira en torno al ciudadano digital, descrito por Hernández et al. 2016 "aquel que utiliza las tecnologías de la información y la comunicación (TICs) con regularidad y eficacia".

Al respecto, una de las competencias transversales de la Universidad Central es precisamente: Establece estrategias comunicativas desde su quehacer personal, como desde su desempeño técnico y profesional a través de diferentes lenguajes y tecnologías de la información, adaptándolos en función de los actores y contextos, a fin de establecer relaciones sociales respetuosas, efectivas y eficaces. Proyecto Educativo y Modelo de Progresión del Estudiante Universidad Central de Chile (2017)

\subsubsection{Juventudes tecnologizadas y uso de TICs en educación superior}

Tal como señalan Garrido et. al. (2016), en la actualidad las juventudes utilizan las TICs en función de sus intereses personales y de sus necesidades de relación social y con fines lúdicos. En definitiva, es posible afirmar que su participación en las redes sociales les aporta capital social y, al mismo tiempo, este capital social influye en la construcción de su identidad y es que los usos de las redes sociales y las TIC juegan un papel importante en las formas de comunicación y en los modos de relacionarse entre la población juvenil lo que se traduce en una oportunidad para la enseñanza virtual que se presenta como una posibilidad atractiva para los estudiantes a través de una instancia en la que el tiempo y el espacio son flexibles, y esto implica, que el protagonista indiscutible es el estudiante, en tanto se responsabiliza por su aprendizaje y su evaluación y, al mismo tiempo, aprende a utilizar recursos diferentes para desarrollar la evaluación de manera autónoma.

Actualmente, los jóvenes tienen un amplio conocimiento y manejo autodidacta de las TICs, que logran compartiendo saberes entre amigos, la mayoría de las veces logran alcanzar un nivel de destreza importante en su uso.

Mark Prensky (citado en Garrido et. al., 2016) sostiene que existe una correlación directa entre el uso de las TIC y la inteligencia de las nuevas generaciones, en la que las juventudes actuales son nativos digitales en tanto han crecido en un entorno tecnológico digital y han interactuado desde su niñez con dichas tecnologías.

Sin embargo, pese a la importancia del uso de las nuevas tecnologías en la vida cotidiana y en el mercado laboral, siguiendo a Garrido, los jóvenes perciben que en los establecimientos educativos no se usan mucho las TICs; más aún, estos nativos digitales se educan en aulas que fueron concebidas para generaciones de jóvenes anteriores que carecían de la «singularidad» del acceso a las TICs.

Al respecto, Santiago et al. (2016) señalan que los medios tecnológicos no se están explotando en todo su potencial en la educación formal, por diversos motivos asociados a la carencia de 
medios técnicos de la organización y al mismo tiempo por la falta de formación en el uso pedagógico de los recursos tecnológicos emergentes con el fin de aprovechar de forma plena sus potencialidades. Este punto es fundamental dado que la incorporación de TICs en el proceso educativo no implica que el docente desaparezca del mismo, sino que más bien lo que ocurre es una transformación de su rol, pues se convierte en un facilitador y un mediador.

Becerril et. al. (2015) señalan que un docente que utiliza TICs en su proceso de enseñanza debe formarse y desarrollar previamente competencias pedagógicas y sociales. En el caso de la evaluación en línea el docente asume diferentes roles en tanto es diseñador de recursos para el estudiante y al mismo tiempo actúa como tutor virtual.

Dentro de las posibilidades tecnológicas aplicadas a la educación, se encuentra el entorno modular dinámico orientado a objetos de aprendizaje Moodle (Modular Object Oriented Dynamic Learning Enviroment) que consiste básicamente en un paquete de software, basado en la idea que el conocimiento se va construyendo por el estudiante a partir de su participación activa en el proceso y en relación con su entorno social, cuyos fundamentos descansan en la pedagogía constructivista social (Casales, Rojas \& Paulí, 2008) y que en la actualidad es una de las plataformas más usadas a nivel mundial en el ámbito universitario.

El objetivo de la plataforma Moodle es facilitar a los docentes las mejores herramientas para gestionar y promover el aprendizaje de sus estudiantes. Dentro de sus ventajas, destacan la facilidad para realizar el seguimiento del trabajo del educando y la ejecución de actividades de retroalimentación y tutoría por parte de los docentes (Pérez et. al., 2009).

Las herramientas que puede utilizar el docente para la promoción del aprendizaje de los estudiantes se subdividen en tres grupos: para generar recursos educativos (editor de cursos, de exámenes, etc.); para seguimiento y evaluación, y para comunicación (en forma asincrónica o sincrónica). Desde el punto de vista de las herramientas de las que disponen los y las estudiantes, se encuentran tres grupos: de formación (visualización de recursos); de seguimiento y evaluación, y de comunicación entre usuarios, de manera sincrónica y asincrónica (Valenzuela et. al., 2013).

En la Universidad Central de Chile, la plataforma Moodle se encuentra operativa desde el año 2005, siendo las primeras carreras en utilizarla las de Ingeniería. El año 2008 se sumaron otras Facultades y el año 2010 la Vicerrectoría Académica implementa la plataforma a nivel corporativo y centralizado como herramienta de apoyo a la docencia. En la actualidad la plataforma es utilizada en la universidad básicamente como repositorio de material bibliográfico y específicamente en la carrera de Trabajo Social, su utilización para la evaluación de los aprendizajes no ha sido abordada, desaprovechando las posibilidades en que, para estos efectos, la plataforma podría ser de utilidad.

\subsubsection{Evaluación en línea}

Lezcano y Vilanova (2017) plantean que la tecnología está cambiando el escenario educativo, al ser herramientas que permiten agilizar, optimizar y extender procesos de enseñanza y aprendizaje. En este sentido, su influencia en los modos de aprender genera nuevos desafíos para la evaluación. Los autores señalan que los cambios en las prácticas, en la forma de desenvolverse de profesores y estudiantes en estos nuevos espacios comunicativos, pueden ser considerados como verdaderos cambios de su rol en los procesos de enseñanza y de aprendizaje. 
Por su parte Dorrego (2006) considera a la evaluación de los aprendizajes como un proceso a través del cual los estudiantes adquieren una comprensión respecto de sus propias competencias y progreso y al mismo tiempo se constituye como un proceso mediante el cual son calificados.

En esta misma línea la Universidad Central de Chile declara que la evaluación "debiera centrarse en ayudar a los estudiantes a aprender de manera que las tareas de evaluación se consideren también como tareas de aprendizaje" (Manual de apoyo docente UCEN, 2017, p. 9).

Siguiendo a Dorrego (2006) el propósito de la evaluación formativa radica en recabar información acerca del progreso de un estudiante para darle retroalimentación a él y a su docente. En este sentido el rol de la retroalimentación es crucial, en tanto permite proporcionar reforzamiento motivacional positivo para el estudiante, lo que se traduce en un mayor compromiso con la asignatura.

La autora señala que el uso de tecnologías para la evaluación de aprendizajes aporta una serie de facilitadores que no están disponibles para el estudiante en las salas de clases constituidas en ambientes tradicionales de la educación superior, como por ejemplo el nivel de inmediatez así como de interacciones; las posibilidades de acceso a las cursos desde cualquier lugar y tiempo ya sea a través del teléfono o de un computador; y la posibilidad de retorno de comentarios y de discusión en instancias como los foros que ayudan a la construcción del aprendizaje por el propio estudiante.

Siguiendo con los facilitadores destaca el ahorro de tiempo en cuanto a desarrollo y distribución, ya que dichas evaluaciones al ser creadas mediante plantillas pueden ser adaptadas y clonadas las veces que se necesite, y así mismo pueden ser implementadas y recolectadas a través del aula virtual, lo que reduce significativamente el tiempo tanto de devolución al estudiante como de corrección. Así mismo destaca la reducción de recursos como papelería y tinta requeridos para la impresión de las evaluaciones en formato tradicional, y facilita los registros de resultados obtenidos por estudiantes, lo que otorga mayor utilidad al uso de los datos.

Respecto de las debilidades de la implementación del proceso de evaluación, Dorrego (2006) destaca que puede ser costosa y consumir mucho tiempo previo para el docente; así mismo es necesario que el hardware y el software sean monitoreados cuidadosamente para evitar fallas durante los exámenes; respecto de los estudiantes éstos requieren poseer o ser entrenados en torno a las habilidades adecuadas a las TICs.

Siguiendo las recomendaciones de Dorrego (2016) respecto de tener en consideración la complejidad del proceso de aprendizaje, la evaluación en línea debe incluir una diversidad de métodos que ofrezcan posibilidades variadas de desarrollo a los estudiantes.

Al respecto, el aula virtual de la Universidad Central es la plataforma Moodle 3.4 que ofrece las siguientes posibilidades de instrumentos evaluativos: Creación de pruebas de selección múltiple, de completación y desarrollo en línea; foros; envío de archivos, creación de portafolios entre otros.

En cuanto al foro, se refiere a una pregunta de debate que se contextualiza con un planteamiento o caso, estableciendo la finalidad y las orientaciones del debate en sí, el cual se irá enriqueciendo con las intervenciones y las experiencias de los estudiantes. La valoración transversal de las intervenciones en el foro, considera la claridad de la comunicación escrita, la capacidad de expresar las ideas, la reflexión personal, el debate de ideas entre los estudiantes, la construcción de argumentos fundamentados considerando el contenido de la unidad y el 
aporte de otros recursos de información relevantes para la temática. En los foros se da la oportunidad a cada estudiante de participar, por lo tanto, la evaluación pasa a ser una instancia de aprendizaje significativo. La mayor riqueza de los foros de discusión es que ofrecen tanto al docente como a los estudiantes la posibilidad de constituir comunidades de aprendizaje.

La tarea consiste en el desarrollo por parte del estudiante de unas instrucciones previamente establecidas por el docente en un archivo dispuesto en el aula virtual, por lo tanto, para acceder a ella el estudiante debe ingresar al aula, descargar dicho archivo, leer comprensivamente y seguir las instrucciones que allí se señalan las que se trabajan en la plantilla en formato Word que luego, una vez desarrollada, el estudiante debe subir y cargar nuevamente al aula virtual.

Por su parte, el control o prueba en línea ofrece la posibilidad de presentar preguntas en distintos formatos: una evaluación de desarrollo o un cuestionario que puede incluir preguntas de alternativa única o múltiples, preguntas de verdadero o falso, y/o preguntas de completación, con un tiempo límite de desarrollo o de entrega. Su propósito es verificar conocimientos, destrezas y habilidades, según las capacidades y nivel que se declare en el programa de la asignatura.

Finalmente, tal como asegura Dorrego (2016), el aprovechamiento de las oportunidades que ofrece la evaluación en línea sólo puede ocurrir si se cuenta, por un lado, con profesores debidamente motivados y capacitados respecto del diseño e implementación de instancias evaluativas en línea y por el otro lado, se debe contar con estudiantes que estén dispuestos a asumir las nuevas responsabilidades en torno a la construcción de su propio aprendizaje y que obviamente exista la disponibilidad y acceso de docentes y estudiantes a los recursos tecnológicos que requiere la educación en línea.

\subsection{Objetivos y pregunta de investigación}

\section{Pregunta de Investigación}

¿Cuáles son las fortalezas y debilidades de la implementación de evaluaciones en línea según la percepción de estudiantes de primer año y profesores de la carrera de Trabajo Social de la Universidad Central de Chile?

\section{Objetivo General}

Analizar las fortalezas y debilidades de la implementación de evaluaciones en línea según la percepción de estudiantes de primer año y profesores de la carrera de Trabajo Social de la Universidad Central de Chile para establecer su viabilidad y utilidad en el proceso de enseñanza aprendizaje de la carrera

\section{Objetivos específicos}

- Identificar las fortalezas de la implementación de evaluaciones de línea según la percepción de estudiantes de primer año y profesores de la carrera de Trabajo Social de la Universidad Central de Chile

- Identificar las debilidades de la implementación de evaluaciones de línea según la percepción de estudiantes de primer año y profesores de la carrera de Trabajo Social de la Universidad Central de Chile 


\section{Metodología}

El comité curricular de la carrera de Trabajo Social sesiona regularmente con la finalidad de diseñar, actualizar, modificar y evaluar el proyecto curricular respectivo para asegurar su calidad y pertinencia. En este escenario y con el reporte de la baja usabilidad de la plataforma como un recurso para el aprendizaje por parte de los docentes de la carrera, surge la iniciativa de implementar un sistema de evaluación en línea que tribute al desarrollo de competencias digitales tanto en profesores como en estudiantes desde una actitud positiva, crítica y realista respecto de las fortalezas y debilidades del uso de la tecnología en el proceso de enseñanza aprendizaje, atendiendo a la necesidad de alfabetizar digitalmente a los estudiantes de trabajo social ya que tal como señala Biminelis (2017) el vínculo con las TIC y con la política pública electrónica ${ }^{4}$ adquiere cada día una presencia más relevante, constituyéndose en un desafío profesional que no puede enfrentarse adecuadamente sin la preparación previa.

Considerando que se trata de un proyecto de innovación educativa, el Comité Curricular estimó pertinente su implementación en un curso de primer año. Así entonces, se implementó en el curso Pobreza y Exclusión Social, que corresponde a la formación disciplinar de Trabajo Social. Se trata de un curso inicial, del dominio de intervención social, que propone la comprensión de conceptos básicos que permitan desarrollar competencias para la actuación en contextos caracterizados por situaciones de vulnerabilidad.

\section{Etapas del proyecto de innovación educativa}

El proyecto de innovación fue estructurado en 4 etapas que contemplaron el diseño del curso, diagnóstico inicial respecto a saberes previos tanto de la asignatura como del uso del aula virtual, implementación, evaluación y transferencia de resultados a la comunidad educativa.

A continuación, se presentan dichas etapas:

\section{Etapa 1. Diseño del curso contenidos curriculares e instrumentos pedagógicos}

En el caso de la cátedra de Pobreza y Exclusión Social, la propuesta de evaluación en línea implicó la completa automatización del proceso evaluativo, es decir fue necesario un trabajo previo a la dictación de la asignatura por parte del equipo docente en el diseño de los instrumentos evaluativos, lo que implicó seleccionar el material bibliográfico por cada una de las unidades del programa de asignatura, creación del material de las clases, selección tanto de los contenidos para la obtención de las situaciones de evaluación, así como la estrategia evaluativa por cada una de las unidades. Una vez determinado lo anterior, correspondió elaborar las instrucciones para los estudiantes y establecer el formato de respuesta con su respectivo puntaje. Creado el material de clases y el instrumento evaluativo, el equipo de docentes trabajó en la revisión y validación tanto disciplinar como pedagógica respecto de la pertinencia, confiabilidad y objetividad de cada una de las evaluaciones en función del programa de asignatura, las unidades y sus resultados de aprendizaje esperados.

\footnotetext{
${ }^{4}$ Se refiere a todos los servicios sociales estatales que actualmente se encuentran digitalizados.
} 
Proceso que se puede observar en el siguiente diagrama:

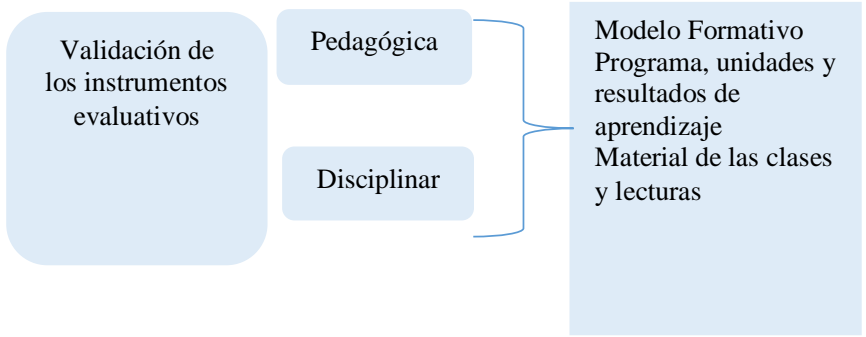

Imagen 1. Diagrama sobre la validación de los instrumentos evaluativos. Fuente: elaboración propia.

Considerando que el propósito de la asignatura es que las y los estudiantes identifiquen diversos enfoques teóricos que sustentan los debates en torno a los conceptos de pobreza y exclusión social, sus formas de medición y su impacto en la formulación de políticas, normativas y estrategias de intervención social, el equipo docente optó por seleccionar tres modalidades que ofrecieran diversos contextos evaluativos considerando el carácter teórico-práctico de la asignatura, así entonces se diseñó como primera evaluación una prueba de selección de alternativas, un foro de discusión y una tarea de desarrollo. La ponderación de las estrategias de evaluación fue la siguiente:

\section{Tabla 1}

Ponderación estrategias de evaluación.

\begin{tabular}{l|l|l|}
\hline $\begin{array}{l}\text { Instancia } \\
\text { evaluativa }\end{array}$ & Unidad & Instrumento \\
\hline $\begin{array}{l}\text { Evaluación } 1 \\
\text { individual }\end{array}$ & I Enfoques de medición de pobreza & Prueba de selección \\
\hline $\begin{array}{l}\text { Evaluación } \\
2 \text { individual }\end{array}$ & $\begin{array}{l}\text { II Pobreza, vulnerabilidad social y } \\
\text { exclusión }\end{array}$ & $\begin{array}{l}\text { Foro aplicación de contenidos } \\
\text { aplico } \\
\text { textos tratados en } \\
\text { clases }\end{array}$ \\
\hline $\begin{array}{l}\text { Evaluación 3 } \\
\text { grupal }\end{array}$ & $\begin{array}{l}\text { III Propuestas de Intervención } \\
\text { desde Trabajo Social }\end{array}$ & \begin{tabular}{l} 
Tarea de desarrollo \\
\hline
\end{tabular}
\end{tabular}

Promedio asignatura

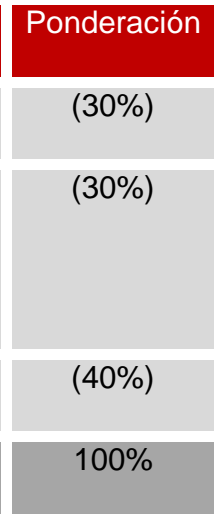

Fuente: elaboración propia.

\section{Etapa 2. Diagnóstico inicial}

Desde la primera clase, en la presentación del syllabus, la docente a cargo del curso advirtió a los estudiantes que las evaluaciones se llevarían a cabo a través del aula virtual Moodle y realizó un sondeo respecto de los principales usos de internet por parte de los estudiantes. Cabe señalar que ninguno de ellos presentó ningún tipo de resistencia o descontento respecto de la modalidad evaluativa. Una de las principales dificultades en esta etapa fue la poca claridad 
de los estudiantes respecto de su clave de acceso al aula virtual ya que ésta es enviada por la Dirección de Gestión Curricular a la dirección de correo institucional el cual no es usado regularmente por los estudiantes.

Durante las 3 primeras clases la docente a cargo mostró al curso las posibilidades de uso del aula virtual y en la clase 4 se implementó una prueba de diagnóstico que se realizaba desde el hogar y correspondió al desarrollo de un cuestionario de 3 preguntas con alternativas respecto de los saberes previos en torno a la asignatura, en esta primera experiencia de un total de 25 estudiantes sólo 19 realizaron la prueba desde sus hogares, por diversos motivos como el no contar con conexión a internet en el hogar ni el celular. Dicha prueba no pudo llevarse a cabo en el laboratorio de la universidad pues ese espacio se encontraba ocupado.

\section{Etapa 3. Implementación}

La etapa de implementación de las evaluaciones planificadas se llevó a cabo conforme se avanzó en las clases expositivas de las correspondientes unidades.

\subsection{Prueba de selección múltiple}

En el caso de la evaluación 1 correspondiente a la prueba de selección múltiple, ésta se llevó a cabo de manera presencial en el laboratorio de computación Vicente Kovacevick 1 de la casa central, y fue temporalizada para estar visible al estudiante en una fecha determinada con un tiempo de 60 minutos para su respuesta.

El instrumento contempló la presentación de 20 preguntas con alternativas y su correspondiente retroalimentación la que era visible para el estudiante una vez dicha prueba había sido finalizada y enviada a calificar.

La retroalimentación es la explicación de la respuesta correcta que se activa por cada pregunta errada, así el estudiante una vez terminada su prueba y enviada a calificar supo al instante y con exactitud en qué preguntas su respuesta no fue correcta y cuáles eran los tópicos que debió considerar y, por supuesto, conoció de manera inmediata la nota obtenida en una escala de 1.0 a 7.0 
A continuación, en el gráfico se muestran los resultados de la primera evaluación:

\section{Resultados evaluación 1}

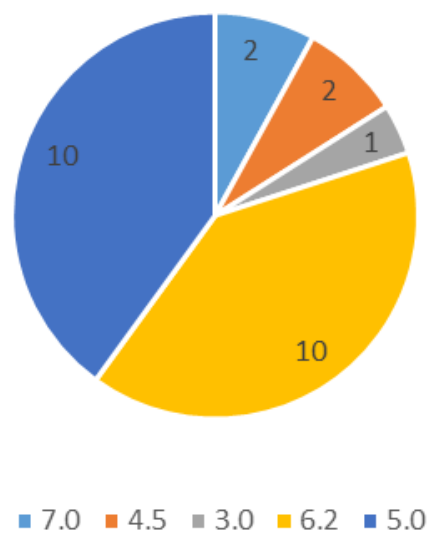

Imagen 2. Gráfico 1 sobre los resultados de la evaluación 1. Fuente: elaboración propia.

Tal como se observa en el gráfico 1, donde se muestran los resultados de la primera evaluación es posible ver que dos estudiantes obtuvieron nota 7.0; 10 de ellos obtuvo nota 5.0; dos tuvieron nota 4.5 y uno tuvo nota 3.0. La escala de notas fue de 60 puntos máximos, cada pregunta valía 3 puntos. La nota 4.0 se obtenía con 36 puntos (en Chile la escala de notas es de 1 a 7 ).

La mayor dificultad en torno a la implementación de esta evaluación se vio reflejada en la lentitud del internet al momento de avanzar a la siguiente pregunta la página demoró al menos 4 minutos en cargar y eso generó bastante ansiedad e inseguridad en los estudiantes porque temían que sus respuestas no se hubieran guardado. Así mismo, al menos 3 estudiantes indicaron que hubiesen preferido tener la posibilidad de responder con texto más que elegir entre alternativas.

La fortaleza estuvo dada en la satisfacción de parte de los estudiantes al conocer la calificación inmediatamente una vez terminada la prueba, así como la retroalimentación en el caso de las respuestas que fueron respondidas de manera errada. "Me voy tranquilo/a" fue la expresión más común entre los estudiantes, una vez revisada la retroalimentación, después del término de la prueba.

\subsection{Foro de Discusión}

En el caso del foro asincrónico de discusión se trabajó mediante la presentación en el aula virtual de 2 preguntas de reflexión en torno a un análisis de caso del trayecto de vida del joven infractor de ley Cristóbal Cabrera apodado por los medios de comunicación como "Cisarro", dicho análisis exigió al estudiante la aplicación del contenido sobre factores de riesgo y protección en el contexto de pobreza y exclusión social. La pauta de indicaciones de evaluación del foro señalaba que la participación contemplaba 2 instancias. En la primera instancia, el estudiante debía responder directamente a la pregunta del foro que solicitaba identificar los factores de riesgo y protección que influyeron en la trayectoria delictiva del joven y en la 
segunda instancia, el estudiante debía comentar la aportación de 1 compañero/a cada, en ambos casos la extensión de los mensajes debía contener un mínimo de 300 palabras. El foro estuvo abierto durante 1 semana y los estudiantes podían responder desde cualquier dispositivo compatible con el aula virtual.

Con el objeto de moldear actitudes y estilos comunicacionales entre la diversidad de estudiantes, la docente a cargo del curso estableció ciertos mínimos esperables en cada comunicación asociados al uso de lenguaje académico y respeto entre compañeros y para con la docente.

Así entonces el presente proyecto buscó utilizar el foro de discusión como instancia de evaluación formativa en la que el estudiante expresa su saber ser a través del dialogo escrito con sus compañeros, las tareas y controles en línea favorecerían el desarrollo de destrezas y habilidades, según las capacidades y nivel que se declara en el programa de la asignatura.

\section{Resultados evaluación 2}

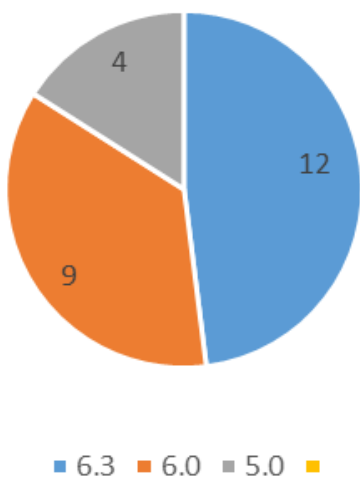

Imagen 3. Gráfico 2 sobre los resultados de la evaluación 2. Fuente: elaboración propia.

Como es posible observar en el gráfico 2, 12 estudiantes obtuvieron nota 6.3, 9 obtuvieron nota 6.0 y 4 obtuvo nota 5.0. En la segunda evaluación de foro: 1. ¿Qué factores de riesgo influyeron en que Cristóbal Cabrera comenzara a temprana edad a delinquir?, 2. ¿Fundamente por qué este joven se encuentra excluido socialmente?

Las instrucciones indicaban al estudiante que además de responder dichas preguntas debía comentar de manera respetuosa y argumentada la respuesta de 1 compañero/a.

En relación a las fortalezas esta fue una de las instancias evaluativas más enriquecedoras de todo el proceso ya que permitió un fluido intercambio de ideas, opiniones y prejuicios respecto de la trayectoria delictiva del joven, si bien hubo posturas diversas, todas fueron expresadas en un tono de respeto y valoración entre compañeros.

Respecto a dificultades en torno a la implementación de esta evaluación cabe señalar que hubo dos estudiantes que no comprendieron la instrucción y enviaron un archivo con sus respuestas, 
frente a ello la docente a cargo agradeció el envío y explicó la importancia de que se respetara el formato de respuestas, ante lo cual los estudiantes reaccionaron positivamente enviando nuevamente su respuesta. En general la mayoría de los estudiantes refirió que les había complicado cumplir con el mínimo de 300 palabras por respuesta, lo que podría deberse a que, al ser estudiantes de primer año, no están acostumbrados al trabajo de argumentación de sus respuestas.

\subsection{Tarea de Desarrollo}

Finalmente, la evaluación 3 correspondió a una tarea de desarrollo grupal que implicó por parte de los estudiantes el desarrollo de una reflexión y análisis grupal en torno al enfoque de pobreza utilizado por los diversos instrumentos de medición y estratificación social en los que se ha basado la política social, desde la FICHA CAS hasta el actual Registro Social de Hogares, y generar una propuesta que pudiera dar respuesta a los actuales escenarios de pobreza en el país. Esta tarea debía ser enviada como archivo al aula virtual.

Se conformaron 6 grupos por afinidad, de cuatro personas 5 grupos de 4 y uno 5 integrantes.

Los resultados fueron los siguientes:

\section{Resultados evaluación 3}

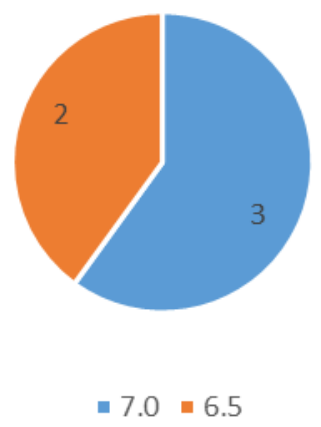

Imagen 4. Gráfico 3 sobre los resultados de la evaluación 3. Fuente: elaboración propia.

Tres grupos obtuvieron nota 7.0 y dos grupos obtuvieron nota 6.5. La tarea contaba con un total de 70 puntos el puntaje de corte para la nota 4.0 fue 35 puntos.

\section{Resultados y análisis}

La investigación se llevó a cabo desde una metodología mixta. Respecto a la riqueza de las investigaciones con enfoque mixto Núñez (2017) plantea que el fundamento central de los métodos mixtos radica en la retroalimentación de los métodos cualitativos y cuantitativos dentro de una perspectiva metodológica única y coherente, que permitiría lograr un nivel de comprensión del objeto investigativo mucho más cercano a la complejidad de fenómeno. 
El curso contó con 25 estudiantes en total, de los cuales 8 eran hombres y 17 mujeres, dado que Trabajo Social es una carrera feminizada, las edades de los estudiantes fluctuaban entre los 19 y 22 años.

\section{Resultados Encuesta de satisfacción}

Al terminar el curso se implementó una encuesta de autoaplicación a través del aula virtual que buscó medir la satisfacción de los y las estudiantes respecto de la metodología de evaluación de la asignatura mediante 23 reactivos de selección escala tipo Likert organizados en torno a las siguientes dimensiones: Aula Virtual, diseño de las evaluaciones, proceso de aprendizaje.

\section{Tabla 2}

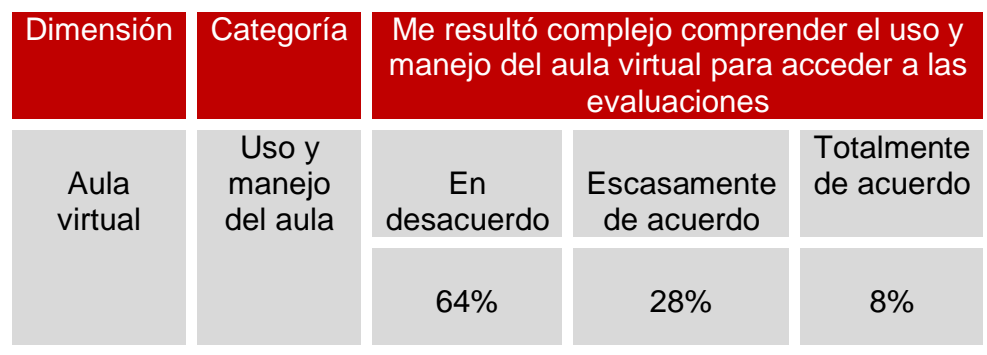

Fuente: elaboración propia con base en encuesta de satisfacción.

Frente a la afirmación: Me resultó complejo comprender el uso y manejo del aula virtual para acceder a las evaluaciones, el $64 \%$ de los estudiantes encuestados respondió estar en desacuerdo, el $28 \%$ escasamente de acuerdo y el $8 \%$ declaró estar totalmente de acuerdo. El resultado sin duda obedece a la familiaridad de los jóvenes nativos digitales respecto del uso diario de las TICs para diversas actividades.

\section{Tabla 2}

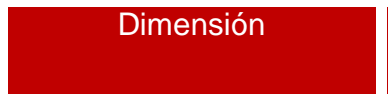

Diseño de las evaluaciones

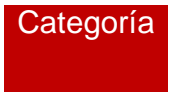

Uso y manejo del aula
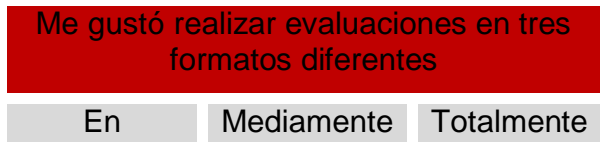
desacuerdo de acuerdo de acuerdo

$4 \%$

$16 \%$ $76 \%$

Fuente: elaboración propia con base en encuesta de satisfacción.

Respecto de la afirmación: Me gustó realizar evaluaciones en tres formatos diferentes, el $76 \%$ de los estudiantes encuestados se manifestó totalmente de acuerdo, el 16\% medianamente de acuerdo y el $4 \%$ en desacuerdo. Recordar que durante el desarrollo de la asignatura se presentaron tres diferentes formatos de evaluación. 
Considerando que es importante que los docentes se aseguren que todos los estudiantes, por un lado, pueden percibir la información clave que se les quiere proporcionar y, por otro lado, puedan expresar de manera diversa lo aprendido atendiendo a los diversos estilos de aprendizaje, resultó fundamental que la asignatura presentara formas alternativas de evaluación que permitieron medir los diferentes tipos de saberes, así entonces en la prueba de selección se midieron principalmente saberes teóricos, en el foro se midieron saberes asociados a la aplicación de contenidos y a lo actitudinal y en la tarea de desarrollo se midieron los tres saberes.

\section{Tabla 3}

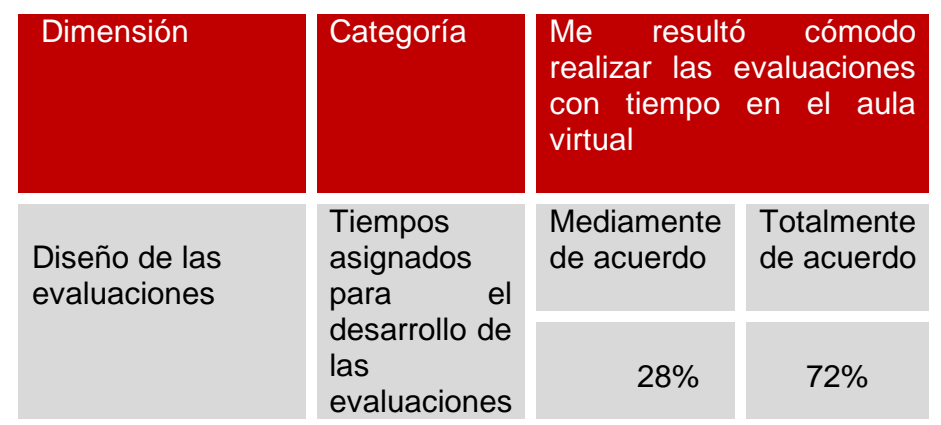

Fuente: elaboración propia con base en encuesta de satisfacción.

Frente a la afirmación "me resultó cómodo realizar las evaluaciones con tiempo en el aula virtual, el $72 \%$ de los estudiantes se manifestó totalmente de acuerdo, mientras el $28 \%$ declaró estar medianamente de acuerdo, esta categoría de análisis resulta de gran importancia ya que desde el equipo docente se pensaba que el agregar tiempo a la prueba podría ser un factor estresor y resultó no ser así para los estudiantes.

\section{Tabla 4}

\begin{tabular}{|c|c|c|c|c|}
\hline Dimensión & Categoría & $\begin{array}{l}\text { Es motivador y } \\
\text { los y las comp }\end{array}$ & $\begin{array}{l}\text { entretenido in } \\
\text { ñeras a través }\end{array}$ & $\begin{array}{l}\text { ractuar con } \\
\text { de los foros }\end{array}$ \\
\hline \multirow{2}{*}{$\begin{array}{l}\text { Diseño de } \\
\text { las } \\
\text { evaluaciones }\end{array}$} & \multirow{2}{*}{$\begin{array}{l}\text { Motivación } \\
\text { frente a la } \\
\text { interacción } \\
\text { en los } \\
\text { foros }\end{array}$} & $\begin{array}{l}\text { Escasamente } \\
\text { de acuerdo }\end{array}$ & $\begin{array}{l}\text { Mediamente } \\
\text { de acuerdo }\end{array}$ & $\begin{array}{l}\text { Totalmente } \\
\text { de acuerdo }\end{array}$ \\
\hline & & $4 \%$ & $28 \%$ & $68 \%$ \\
\hline
\end{tabular}

Fuente: elaboración propia con base en encuesta de satisfacción.

Es motivador y entretenido interactuar con los y las compañeras través de los foros, el $68 \%$ de los estudiantes se manifestó totalmente de acuerdo, mientras el $28 \%$ declaró estar medianamente de acuerdo y un $4 \%$ señaló estar escasamente de acuerdo con la afirmación.

El componente emocional asociado a la motivación del estudiante es un elemento crucial en el aprendizaje que se pone en evidencia al ver las diferencias en lo que motiva a los estudiantes o 
en la manera en que se implican para aprender. El formato de participación y evaluación a través del foro significó variar las dinámicas comunicacionales y relacionales entre los compañeros, de modo que todos los estudiantes debieron comunicarse bajo los códigos del lenguaje escrito, autorregulándose respecto de los comentarios dirigidos hacia los otros compañeros. En este sentido fue crucial para la docente establecer un clima de apoyo y aceptación en el aula virtual.

\section{Tabla 5}

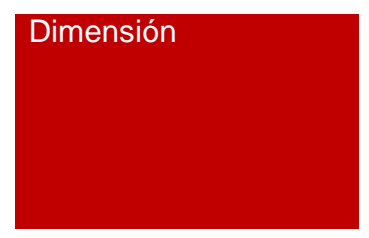

Proceso de
Aprendizaje

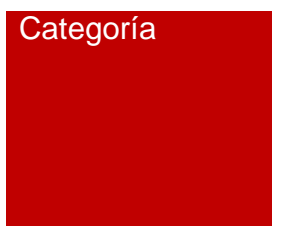

Motivación frente a la retroalimentación inmediata en la prueba de selección
Fue motivador conocer

las calificaciones

obtenidas en la prueba de

selección de manera

automática

Mediamente Totalmente

de acuerdo de acuerdo

$12 \%$

Fuente: elaboración propia con base en encuesta de satisfacción.

Ante la afirmación: fue motivador conocer las calificaciones obtenidas en la de manera automática el $88 \%$ de los estudiantes encuestados señaló estar totalmente de acuerdo demostrando que para los estudiantes resulta fundamental conocer de manera oportuna la calificación obtenida por su desempeño.

\section{Tabla 6}

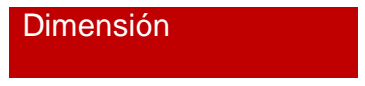

Proceso de Aprendizaje

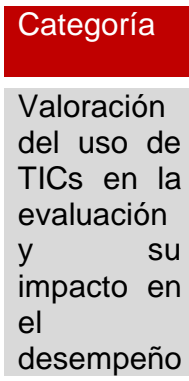

Pienso que la metodología de evaluación afectó positivamente mi desempeño

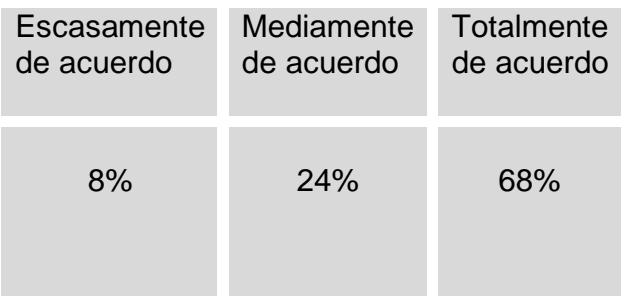

Fuente: elaboración propia con base en encuesta de satisfacción.

Frente a la afirmación: pienso que la metodología de evaluación afectó positivamente mi desempeño el $68 \%$ de los estudiantes encuestados señaló estar totalmente de acuerdo lo que se condice con el aumento sostenido de las calificaciones obtenidas en las tres instancias evaluativas. 


\section{Resultados Grupo Focal}

Considerando que el curso en sí mismo constituía un grupo social se realizaron dos grupos focales durante el proceso de implementación de la evaluación en línea

\section{a. Desde la percepción de los estudiantes} Fortalezas:

- La retroalimentación y calificación inmediatas que ofreció la prueba de selección múltiple, de acuerdo a los resultados obtenidos, fue uno de los factores que fomentó la motivación con la asignatura ya que se constituyeron en un refuerzo oportuno respecto de los elementos que como estudiantes debían fortalecer.

- Así también resultó motivador para los estudiantes la interacción a través del foro entre compañeros tanto por el formato de evaluación que se basó en el análisis del caso real de un joven infractor de ley chileno, como porque en torno al análisis y aplicación del contenido de la asignatura en dicho caso pudieron compartir aprendizajes y opiniones diversas frente a un determinado tema, así fue señalado en el grupo focal de estudiantes:

"En los foros igual no se trataba de copiar y pegar, hubo que aplicar los contenidos y eso fue entretenido porque pudimos analizar la historia del Cisarro como profesionales" Grupo Focal estudiantes.

- La posibilidad de acceso al aula virtual y por ende a la asignatura y sus recursos desde cualquier dispositivo y desde cualquier lugar físico fue reconocida como una fortaleza, en este sentido la posibilidad de realizar las evaluaciones desde los hogares fue señalada como una fortaleza en el sentido de que permitía a cada estudiante controlar el entorno físico y el ambiente para disponerse a responder la evaluación. Así mismo fue considerada una fortaleza el hecho de que los recursos de la asignatura estuvieron disponibles en el aula virtual durante todo el curso. Al respecto, señalaron: "los materiales siempre estuvieron ahí, no hubo que andar detrás del profesor pidiéndole las cosas" (Grupo Focal estudiantes).

- Otra de las fortalezas reconocidas por los estudiantes se relaciona con la pertinencia de la metodología evaluativa de la asignatura con la actual sociedad del conocimiento, por lo que fue recocida como una instancia que permitió desplegar la habilidad del uso de TICs necesaria para el Trabajo Social actual.

\section{Debilidades:}

- Una de las debilidades reconocidas por los estudiantes tuvo relación con que el proceso de asignación de las claves de acceso al aula virtual, por parte de la universidad, resultó engorroso.

- No todos los estudiantes se reconocen como nativos digitales, por ende, no todos tienen expertiz en el uso de las TICs, por lo que para un par de estudiantes la experiencia resultó compleja, pero de igual modo la consideraron importante en función de la importancia que tiene el uso de TICs en el mundo laboral.

- Otra de las debilidades señaladas por los estudiantes es que no todos disponen de conexión a internet en sus casas y realizar las evaluaciones desde el celular no les daba confianza porque sus planes de celular al ser básicos podían ser más lentos. 
- Así mismo los estudiantes señalaron que particularmente las pruebas de selección de alternativas aplican sólo a determinados tipos de contenidos específicos por lo que su aplicación sería limitada.

b. Desde la percepción de los profesores a cargo del proyecto Fortalezas:

- Disposición de los estudiantes frente a la modalidad evaluativa. En este sentido la docente a cargo comenta: "una de las razones por las cuales desde el Comité Curricular se decide la implementación del proyecto en un curso de primer año, tuvo mucho que ver con que el estudiante de primer año viene con otra disposición, su motivación de igual manera es mayor, entonces resultó bastante grato el trabajo con ellos "(Grupo Focal docentes).

- La rapidez en la calificación de la prueba de selección múltiple otorgó una mayor facilidad al uso de los datos por lo que el traspaso de las calificaciones por cada estudiante resultó más práctico que la calificación manual.

- Ahorro en el uso de papel e impresiones.

- Menor tiempo en el desarrollo de las respuestas por parte de los estudiantes.

- Los docentes destacaron que instancias como los foros ayudan a la construcción del aprendizaje por el propio estudiante y al mismo tiempo fortalece el lenguaje escrito y la capacidad de debate y fundamentación de una idea.

\section{Debilidades:}

- La etapa de diseño del curso, así como de sus contenidos curriculares e instrumentos pedagógicos implica un arduo trabajo previo a la dictación del curso.

- La plataforma colapsó el día que todos los estudiantes realizaron la prueba de selección múltiple en línea de manera sincrónica lo que enlenteció el avance de la evaluación y generó cierta inseguridad en los estudiantes respecto de si se habían o no guardado sus respuestas.

- Otra de las debilidades detectadas por el equipo docente tiene que ver con la dificultad para acceder a la agenda de laboratorios de computación por la alta demanda que tienen estos.

- Desconfianza de los profesores de las otras secciones frente a la posibilidad de que el estudiante copie si realiza las pruebas en línea desde otros espacios fuera del horario de clases.

- El equipo docente no dispuso del tiempo necesario para la elaboración de un instructivo acerca de cada instrumento evaluativo y el paso a paso para el estudiante por lo que las instrucciones en torno al uso del aula virtual se explicaron en la sala de clases, pero de manera verbal.

- Sólo una de las docentes del equipo a cargo del proyecto conocía y manejaba las posibilidades de usabilidad del Moodle para la creación de pruebas de selección múltiple. 


\section{Conclusiones y Discusión}

La implementación de evaluaciones en línea al interior de una cátedra de primer año es una iniciativa que se realiza por primera vez en la carrera de Trabajo Social de la Universidad Central, y su relevancia y contribución radica en que permite familiarizar a los estudiantes con el uso de TICs, entendida como habilidad fundamental para el actual mundo laboral, de manera que logren incorporar en su formación y en el ejercicio profesional el uso de las tecnologías de la información y la comunicación con regularidad y eficacia, tarea que según Prensky no resulta compleja para las juventudes estudiantes, en tanto nativos digitales, y al mismo tiempo, se constituyó en una instancia de innovación académica.

Respecto de las fortalezas de la implementación de evaluaciones en línea según la valoración de los estudiantes, los análisis cuantitativos de los resultados del proyecto indicaron que, en general, a los estudiantes no les resultó complejo comprender el uso y manejo del aula virtual, aun cuando en el grupo focal reconocieron que la asignación de las claves de acceso a dicha aula por parte de la universidad resultó un tanto engorroso. Los resultados cuantitativos y cualitativos de las tres evaluaciones implementadas en la cátedra permiten afirmar que los estudiantes se responsabilizaron por su proceso de aprendizaje y evaluación, y aprendieron efectivamente a utilizar recursos diferentes para desarrollar las evaluaciones a través del aula virtual de manera autónoma y con un rendimiento académico óptimo. Desde aquí entonces es posible afirmar que la evaluación en línea es absolutamente pertinente y viable no sólo en cursos de primer año sino en todos los niveles de la carrera porque tanto estudiantes como docentes evidenciaron más fortalezas que debilidades respecto de su implementación entre las que se cuenta el abaratar costos de papelería e impresión, menor tiempo en el desarrollo de las evaluaciones, menor tiempo en el proceso de calificación en el caso de las pruebas de selección, acceso a los recursos desde cualquier dispositivo y lugar.

Cabe destacar la actitud positiva de los estudiantes frente a la modalidad evaluativa sin la cual el proyecto no hubiese tenido impacto, así mismo se destaca el trabajo realizado por los docentes en cuanto al proceso de diseño instruccional ya que el diseño de las evaluaciones ocupó un papel preponderante en la cátedra tanto en sus propósitos formativo y sumativo.

Respecto de la evaluación en línea los estudiantes se manifestaron de acuerdo en su implementación pues señalaron reconocer la importancia de adquirir el uso y manejo de TICs en el proceso de aprendizaje atendiendo a la nueva figura del ciudadano digital.

Por su parte la retroalimentación inmediata en la prueba de selección múltiple, contribuyó de manera importante en la motivación de los estudiantes con la asignatura y eso se pudo ver reflejado en el permanente porcentaje de asistencia durante el semestre el cual no bajó del $90 \%$, en este punto el equipo docente coincide en que el uso de tecnologías para la evaluación de aprendizajes aporta una serie de facilitadores que de acuerdo con Dorrego (2006) no están disponibles para el estudiante en las salas de clases constituidas en ambientes tradicionales.

La participación en el foro en torno a un caso de análisis del joven infractor de ley conocido mediáticamente como "Cisarro" resultó ser una experiencia alentadora, motivante, que contribuyó al establecimiento de confianza por parte de los estudiantes, pues estos fueron construyendo sus aprendizajes a partir de sus propios conocimientos, ampliándolos y fortaleciéndolos tanto con los aportes de la docente a cargo de la asignatura, como con los de los compañeros, toda vez que el foro se constituyó en una comunidad de aprendizaje en la que el tema permitió trabajar no sólo la aplicación de contenidos sino también la deconstrucción de prejuicios en torno al bullado caso del joven infractor de Ley. Otra bondad del foro es que favoreció la comunicación escrita basada en el respeto de la diferencia de género, de religión y 
orientación política de los participantes, en concordancia con los valores institucionales de libertad, tolerancia y dignidad.

Desde dicha experiencia, es posible afirmar que los espacios de foros de discusión en el aula virtual son una excelente herramienta que favorece la evaluación formativa, puesto que, mediante la participación activa en el mismo, el docente tiene la oportunidad de ir aclarando contenidos y orientar a los estudiantes en la elaboración de ideas y argumentos antes de establecer la calificación, dándoles la oportunidad de introducir mejorar en sus respuestas.

Respecto de las debilidades. Efectivamente, tal como expresa Santiago et. al. (2016) en la Universidad Central y, particularmente en la carrera de Trabajo Social, los medios tecnológicos no se están explotando en todo su potencial por diversos motivos, entre los que destaca la falta de formación docente para el uso pedagógico de los recursos tecnológicos emergentes para aprovechar de forma plena sus potencialidades.

En este sentido los hallazgos de la implementación del proyecto de innovación educativa se constituyen en un aporte al trabajo del comité curricular de la carrera ya que dejan en evidencia las posibilidades de réplica en los distintos niveles por su incidencia positiva en la motivación del estudiante y, al mismo tiempo, dan cuenta de la necesidad de generar instancias formativas que tributen al desarrollo de competencias digitales en el cuerpo docente.Tanto docentes como estudiantes requieren mayor formación en Alfabetización digital Proceso de enseñanza y aprendizaje acerca de la tecnología y su uso. Formación y desarrollo de competencias digitales que son claves para los desafíos y el aprovechamiento real de las oportunidades del mundo digital.

"La ciudadanía digital se ha convertido en una de las esferas, cada vez más extendida, de nuestra actividad y participación como ciudadanos del mundo global, por lo que es fundamental incluirla en los programas de educación para la ciudadanía" (MINEDUC, 2019, p. 7).

El análisis de las fortalezas versus las debilidades de la implementación de evaluaciones en línea según la percepción de estudiantes de primer año y profesores de la carrera de Trabajo Social muestra que su implementación es viable y es al mismo tiempo útil en el proceso de enseñanza aprendizaje de la carrera considerando el desarrollo imparable de tecnologías digitales han transformado el mundo laboral y de paso también el contexto del proceso educativo, por lo que educar en la era digital implica desafíos nuevos e importantes que los docentes debemos ir asumiendo, ya que sin duda como señala MINEDUC 2019 las TICs potencian habilidades y competencias propias del siglo XXI que los jóvenes ejercitan en su vida cotidiana, hay que aprovechar esa expertiz y llevarla al ámbito educativo no sólo para que el proceso de enseñanza aprendizaje sea más atractivo sino porque hoy más que nunca dicha práctica se ha convertido en una necesidad, es el gran desafío que tenemos los docentes. Sin embargo, es necesario que exista voluntad y apoyo desde el ámbito institucional para que el cuerpo docente acceda a cursos de actualización digital y uso de TICs en el proceso formativo. Considerando que la tecnología está cambiando el escenario laboral y educativo Lezcano y Vilanova (2017) plantean que resulta coherente utilizarla como herramienta para agilizar, optimizar y extender procesos de enseñanza y aprendizaje. 


\section{Referencias}

Becerril C., Sosa G., Delgadillo M. y Torres S. (2015). Competencias Básicas de un Docente Virtual. Revista de Sistemas y Gestión Educativa, 2 (4), 882-887. Recuperado de: https://ecorfan.org/bolivia/researchjournals/Sistemas_y_Gestion_Educativa/vol2num4/1 8.pdf

Binimelis-Espinoza, Helder. (2017). Gobierno electrónico como tecnología de inclusión social. Reflexiones desde el Trabajo Social. Revista Katálysis [en linea], 20 (3), 448-457. Recuperado de: https://www.redalyc.org/articulo.oa?id=179653096014

Casales, R., Rojas, J. y Paulí, G. (2008). Algunas experiencias didácticas en el entorno de la plataforma Moodle. Revista de Informática Educativa y Medios Audiovisuales, 5 (19), 1 10. Recuperado

de: http://laboratorios.fi.uba.ar/lie/Revista/Articulos/050510/A1mar2008.pdf

Dorrego, E. (2006). Educación a Distancia y Evaluación del Aprendizaje. RED. Revista de Educación a Distancia. 50 (12). Recuperado de: http://www.um.es/ead/red/M6/dorrego.pdf

Garrido M.; Busquet J.; Munté R. (2016). De las TIC a las TRIC. Estudio sobre el uso de las TIC y la brecha digital entre adultos y adolescentes en España. Anàlisi. Quaderns de Comunicación y Cultura, 54, 44-57. Recuperado de: http://dx.doi.org/10.7238/a.v0i54.2953

Hernández Suárez, C., Ayala García, E., Gamboa Suárez, A. (2016). Modelo de competencias TIC para docentes: Una propuesta para la construcción de contextos educativos innovadores y la consolidación de aprendizajes en educación superior. Revista Katharsis, 22, 221-265. Recuperado de: http://revistas.iue.edu.co/index.php/katharsis

Lezcano L. y Vilanova, G. (2017). Instrumentos de evaluación de aprendizaje en entornos virtuales. Perspectiva de estudiantes y aportes de docentes. Unidad Académica Caleta Olivia. Argentina: Universidad Nacional de la Patagonia Austral. Recuperado de: Dialnet-InstrumentosDeEvaluacionDeAprendizajeEnEntornosVir-5919087\%20(2).pdf

Mineduc. (2019). Ciudadanía Digital Recuperado de: https://formacionciudadana.mineduc.cl/wpcontent/uploads/sites/46/2019/06/CiudadaniaDigital-Cuadernillo.pdf

Núñez J. (2017). Los métodos mixtos en la investigación en educación: Hacia un uso reflexivo. Recuperado de: http://dx.doi.org/10.1590/198053143763

Pérez, M.T.; Martín, M.; Arratia, O. y Galisteo, D. (2009). Innovación en docencia universitaria con Moodle: Casos prácticos. San Vicente, Alicante: Editorial Club Universitario.

Santiago R.; Navaridas F.; Andía L.A. (2016). Las percepciones de los directivos de centros escolares sobre el uso y el valor de las TIC para el cambio e innovación educativa. Estudios sobre Educación, 30, 145-174. Recuperado de: https://dadun.unav.edu/bitstream/10171/40345/1/7.\%20A.\%20Ra\%c3\%bal\%20Santiag 0\%20Campi\%c3\%b3n.pdf

Universidad Central de Chile. (2017). Manual de Apoyo Docente. Santiago de Chile: Dirección de Calidad Educativa, Vicerrectoría Académica.

Valenzuela-Zambrano, B. y Pérez-Villalobos, M. V. (2013). Aprendizaje autorregulado a través de la plataforma virtual Moodle. Educación y Educadores, 16 (1), 66-79. 